\title{
THE PENNY-SHAPED CRACK IN SHEAR AND RELATED CONTACT PROBLEMS
}

\author{
J. R. BARBER
}

Department of Mechanical Engineering, University of Newcastle upon Tyne, Newcastle upon Tyne, NE1 7RU, England

\begin{abstract}
A solution to the equations of elastic equilibrium is developed in terms of two harmonic functions, such that on a certain plane, normal stresses are identically zero, whilst tangential stresses and displacements are expressible as simple derivatives.

The solution is used to solve the problem of the penny-shaped crack subjected to an arbitrary tangential traction and the corresponding contact problem, using a method developed by Green.

The method is applied by way of illustration to Mindlin's problem of the tangential compliance of elastic spheres in contact and to the penny-shaped crack in uniform shear and in torsion about a diametral axis.
\end{abstract}

\section{INTRODUCTION}

IN A PREVIOUS paper[1], it was demonstrated that, if the tangential tractions and displacements at the surface of an elastic half-space are expressed in terms of two-dimensional stress and displacement potentials, the relation between these potentials is mathematically analogous with that between normal traction and displacement at the surface of the half-space. This suggests that it might be possible to develop a solution to the three-dimensional equations of elastic equilibrium in terms of harmonic functions which reduce to suitable two-dimensional potentials on the surface plane.

Such a solution is here developed and applied to the problem of the penny-shaped crack in non-axisymmetric shear and the corresponding contact problem, using an extension of the method due to Green [2]. The method is illustrated by application to Mindlin's problem of the tangential compliance of two elastic spheres in contact and to the penny-shaped crack in uniform shear and in torsion about a diametrical axis.

The method compares very favourably in mathematical simplicity with the previous solution to this class of problems due to Westmann [3] who made use of the asymmetric solution of the equations of elastic equilibrium in terms of Hankel transforms developed by Muki [4]. This latter method has recently been used by Lowengrub and Sneddon [5] in the treatment of the more complex problem of shear of a half-space imperfectly bounded to a rigid foundation.

\section{THE GENERAL SOLUTION}

A suitable solution to the three dimensional equations of elastic equilibrium can be obtained by superposing solutions $A, B, E$ of Green and Zerna ([6], pp. 169-171) and applying the condition that the normal traction should be zero on the surface $z=0$. The semi-infinite solid is regarded as occupying the space $z \geq 0$ in rectangular cartesian co-ordinates $x, y, z$ or cylindrical polar co-ordinates $r, \theta, z$.

The displacement vector $\mathbf{u}$ can be expressed in the form

$$
\mathbf{u}=\nabla \phi+\frac{1}{2(1-\nu)}(z \nabla-(3-4 \nu) \mathbf{k}) \frac{\partial \phi}{\partial z}+\operatorname{curl} \mathbf{k} \psi
$$

where $\nabla^{2} \phi=\nabla^{2} \psi=0$ 
The stress component $\mathbf{s}_{z}$ acting on planes perpendicular to the $z$ axis is

$$
\mathbf{s}_{z}=\frac{G}{(1-\nu)} \frac{\partial}{\partial z}(z \nabla-\mathbf{k}) \frac{\partial \phi}{\partial z}+G \operatorname{curl} \mathbf{k} \frac{\partial \psi}{\partial z}
$$

whilst the components in the $z$ plane are conveniently represented in terms of the complex variable $\xi=x+i y=r \mathrm{e}^{i \theta}$ and its conjugate $\bar{\xi}$ in the form

$$
\left.\begin{array}{rl}
\sigma_{x x}+\sigma_{y y}=\sigma_{r r}+\sigma_{\theta \theta} & =\frac{-G}{(1-\nu)}\left(2(1+\nu)+\frac{z \partial}{\partial z}\right) \frac{\partial^{2} \phi}{\partial z^{2}} ; \\
\sigma_{x x}+2 i \sigma_{x y}-\sigma_{y y} & =\mathrm{e}^{2 i \theta}\left(\sigma_{r r}+2 i \sigma_{r \theta}-\sigma_{\theta \theta}\right) \\
& =4 G \frac{\partial^{2}}{\partial \bar{\xi}^{2}}\left(2(\phi-i \psi)+\frac{z}{(1-\nu)} \frac{\partial \phi}{\partial z}\right),
\end{array}\right\}
$$

where $G, \nu$ are respectively the modulus of rigidity and Poisson's ratio for the material.

On the surface $z=0$, we have

$$
\left.\begin{array}{c}
u_{x}+i u_{y}=\mathrm{e}^{i \theta}\left(u_{r}+i u_{\theta}\right)=\frac{2 \partial}{\partial \dot{\xi}}(\phi-i \psi) \\
u_{z}=-\frac{(1-2 \nu)}{2(1-\nu)} \frac{\partial \phi}{\partial z}
\end{array}\right\}
$$

It follows that surface values of the functions $\phi, \psi$ or $\frac{\partial \phi}{\partial z}, \frac{\partial \psi}{\partial z}$ can be obtained from given surface displacements or tractions respectively, by means of the equations

$$
\begin{aligned}
4 \frac{\partial^{2}}{\partial \xi \partial \xi}(\phi-i \psi) & =-\frac{\partial^{2}}{\partial z^{2}}(\phi-i \psi)=2 \frac{\partial}{\partial \xi}\left(u_{x}+i u_{y}\right) \\
4 G & \frac{\partial^{2}}{\partial \xi \partial \xi}\left(\frac{1}{(1-\nu)} \frac{\partial \phi}{\partial z}-\frac{i \partial \psi}{\partial z}\right)=G \frac{\partial^{3}}{\partial z^{3}}\left(\frac{1}{(1-\nu)} \frac{\partial \phi}{\partial z}-\frac{i \partial \psi}{\partial z}\right) \\
& =\frac{2 \partial}{\partial \xi}\left(\sigma_{x z}+i \sigma_{y z}\right) .
\end{aligned}
$$

Contact and crack problems involving shear stresses only on the surface or crack plane can therefore be resolved into the search for two harmonic functions $\phi, \psi$ having prescribed values on part of the boundary $z=0$, on the rest of which the derivatives $(\partial \phi / \partial z)(\partial \phi / \partial z)$ are known.

\section{THE PENNY-SHAPED CRACK AND CONTACT PROBLEMS}

To apply the above solution to the crack and contact problems, we consider terms in $\phi, \psi$ of the form $\Phi(r, z) \cos n\left(\theta+\theta_{0}\right), \Psi(r, z) \sin n\left(\theta+\theta_{0}\right)$ respectively. More general stress distributions can be expressed as the sum of a series of such functions. 
The boundary conditions to be satisfied on the plane $z=0$ are as follows:-

(a) Contact problem

We assume tangential displacements are known throughout the circular area $r \leq a$ and hence we have

$$
\left.\left.\begin{array}{rl}
\sigma_{r z} & =\sigma_{\theta z}=0, r>a ; \\
\sigma_{z z} & =0, \text { all } r \\
u_{r} & =u_{1}(r) \cos n\left(\theta+\theta_{0}\right), \\
u_{\theta} & =u_{2}(r) \sin n\left(\theta+\theta_{0}\right),
\end{array}\right\} a \geq r\right\}
$$

where $u_{1}, u_{2}$ are prescribed functions of $r$ only. The corresponding boundary conditions on $\Phi, \Psi$ can be found by expressing equations $[5,6]$ in polar co-ordinates and substituting for displacements and stresses from equation [7] to give

$$
\left.\begin{array}{l}
\frac{\partial^{3} \Phi(r, 0)}{\partial z^{3}}=\frac{\partial^{3} \Psi(r, 0)}{\partial z^{3}}=0, r>a \\
\frac{\partial^{2} \Phi(r, 0)}{\partial z^{2}}=-\left\{\frac{\mathrm{d} u_{1}}{\mathrm{~d} r}+\frac{u_{1}+n u_{2}}{r}\right\}, a \geq r \\
\frac{\partial^{2} \Psi(r, 0)}{\partial z^{2}}=\frac{\mathrm{d} u_{2}}{\mathrm{~d} r}+\frac{u_{2}-n u_{1}}{r}, a \geq r .
\end{array}\right\}
$$

Since $\phi, \psi$ and its derivatives with respect to $z$ are harmonic functions, we can restate these conditions in the form

$$
\begin{gathered}
\frac{\partial \Phi(r, 0)}{\partial z}=h_{1}(r), \quad \frac{\partial \Psi(r, 0)}{\partial z}=h_{2}(r), r>a ; \\
\Phi(r, 0)=f(r), \quad \Psi(r, 0)=g(r), a \geq r ;
\end{gathered}
$$

where $h_{1}(r), h_{2}(r)$ are integrals of the equation

$$
\frac{\mathrm{d}^{2} h}{\mathrm{~d} r^{2}}+\frac{1}{r} \frac{\mathrm{d} h}{\mathrm{~d} r}-\frac{n^{2} h}{r^{2}}=0
$$

$f(r)$ is an integral of

$$
\frac{\mathrm{d}^{2} f}{\mathrm{~d} r^{2}}+\frac{1}{r} \frac{\mathrm{d} f}{\mathrm{~d} r}-\frac{n^{2} f}{r^{2}}=\frac{\mathrm{d} u_{1}}{\mathrm{~d} r}+\frac{u_{1}+n u_{2}}{r}
$$

and $g(r)$ is an integral of

$$
\frac{\mathrm{d}^{2} g}{\mathrm{~d} r^{2}}+\frac{1}{r} \frac{\mathrm{d} g}{\mathrm{~d} r}-\frac{n^{2} g}{r}=-\left(\frac{\mathrm{d} u_{2}}{\mathrm{~d} r}+\frac{u_{2}-n u_{1}}{r}\right)
$$

The arbitrary constants in these integrals are determined by considerations of continuity at $r=0, a, \infty$ and by substitution into equations $(3,4,7)$. 
(b) Crack problem

The crack is assumed to extend over the circle $r \leq a$ in the plane $z=0$. Hence we have

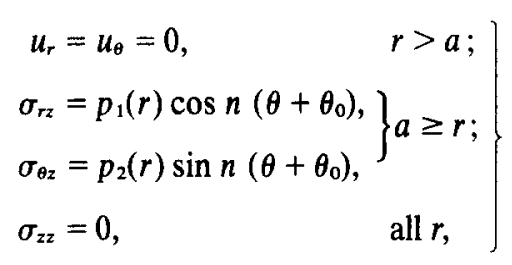

where $p_{1}, p_{2}$ are prescribed functions of $r$ only.

We define further harmonic functions $\mathscr{F}(r, z) \cos n\left(\theta+\theta_{0}\right), \mathscr{G}(r, z) \sin n\left(\theta+\theta_{0}\right)$ by the relations

$$
\frac{\partial \mathscr{F}}{\partial z}=\Phi ; \quad \frac{\partial \mathscr{G}}{\partial z}=\Psi
$$

in terms of which equation (13) can be restated as

$$
\left.\begin{array}{c}
\frac{\partial \mathscr{F}(r, 0)}{\partial z}=h_{1}(r), \frac{\partial \mathscr{G}(r, 0)}{\partial z}=h_{2}(r), r>a ; \\
\mathscr{F}(r, 0)=f(r), \quad \mathscr{G}(r, 0)=g(r), a \geq r,
\end{array}\right\}
$$

where $h_{1}(r), h_{2}(r)$ are integrals of equation $(10), f(r)$ is an integral of

$$
\left(\frac{\mathrm{d}^{2}}{\mathrm{~d} r^{2}}+\frac{1}{r} \frac{\mathrm{d}}{\mathrm{d} r}-\frac{n^{2}}{r^{2}}\right)^{2} f=-\frac{(1-\nu)}{G}\left(\frac{\mathrm{d} p_{1}}{\mathrm{~d} r}+\frac{p_{1}+n p_{2}}{r}\right),
$$

and $g(r)$ is an integral of

$$
\left(\frac{\mathrm{d}^{2}}{\mathrm{~d} r^{2}}+\frac{1}{r} \frac{\mathrm{d}}{\mathrm{d} r}-\frac{n^{2}}{r^{2}}\right)^{2} g=\frac{1}{G}\left(\frac{\mathrm{d} p_{2}}{\mathrm{~d} r}+\frac{p_{2}-n p_{1}}{r}\right) .
$$

\section{METHOD OF SOLUTION}

The boundary conditions as expressed in equations (9.15) are of similar form for both contact and crack problems and the solution can be found using an adaptation of the method developed by Copson [7] and Green[2].

It is convenient to consider each of the functions $\phi, \psi$ as the sum of two harmonic functions, one of which is continuously differentiable across $r=a$ on $z=0$, whilst the other tends to zero on $r>a, z=0$. For example, the boundary conditions on $\Phi$ given in equation (9) become

$$
\left.\begin{array}{lrl}
\frac{\partial \Phi_{1}(r, 0)}{\partial z}=h_{1}(r), & \text { all } r>0 \\
\frac{\partial \Phi_{2}(r, 0)}{\partial z}=0, & r>a \\
\Phi_{2}(r, 0)=f(r)-\Phi_{1}(r, 0), & a \geq r
\end{array}\right\}
$$


The boundary conditions on $\Phi_{1}$ are not mixed and hence we can find $\Phi_{1}(r, 0)$ directly by integration. For example, from equation (5) of Copson's paper [7] we obtain

for all $r>0$.

$$
\Phi_{1}(r, 0)=\frac{-2}{\pi r^{n}} \int_{0}^{r} \frac{t^{2 n}}{\sqrt{ }\left(r^{2}-t^{2}\right)} \int_{t}^{\infty} \frac{h_{1}(s) s^{1-n} \mathrm{~d} s \mathrm{~d} t}{\sqrt{ }\left(s^{2}-t^{2}\right)} .
$$

The boundary conditions on $\Phi_{2}$ are now of the form required for Green's solution and we have

$$
\frac{\partial \Phi_{2}(r, 0)}{\partial z}=\frac{2 r^{n-1}}{\pi} \frac{\mathrm{d}}{\mathrm{d} r} \int_{r}^{a} \frac{t^{1-2 n}}{\sqrt{ }\left(t^{2}-r^{2}\right)} \frac{\mathrm{d}}{\mathrm{d} t}\left\{\int_{0}^{t} \frac{s^{n+1} \Phi_{2}(s, 0) \mathrm{d} s}{\sqrt{ }\left(t^{2}-s^{2}\right)}\right\} \mathrm{d} t
$$

for $a \geq r$, from equations $2.15,2.16$ of reference [2].

We now have values of the derivative $\partial \Phi(r, 0) / \partial z$ for all values of $r$ and the solution can be completed by integration. Alternatively, we can make use of a device developed by Love [8] and Green [2] which permits a more straightforward, though less automatic, solution. The latter method is adequately described in the cited references and it will suffice here to give a brief summary of the notation and procedure.

We define complex conjugate functions $R_{1}, R_{2}$ by the equations

$$
R_{2}=\bar{R}_{1}=\rho \mathrm{e}^{v / 2},(\rho \geq 0) ;
$$

where

$$
\left.\begin{array}{l}
\rho^{2} \cos v=r^{2}+z^{2}-a^{2} \\
\rho^{2} \sin v=2 z a
\end{array}\right\} \pi \geq v \geq 0
$$

These functions tend to $z-i a, z+i a$ respectively on the $z$ axis $(r=0)$, and on the plane $z=0$ we have

$$
\left.\begin{array}{ll}
R_{2}=R_{1}=\left(r^{2}-a^{2}\right)^{1 / 2}, & r>a \\
R_{2}=-R_{1}=i\left(a^{2}-r^{2}\right)^{1 / 2}, & a \geq r
\end{array}\right\}
$$

With this notation, if $f(R, z, \theta)$ defines a real harmonic function in cylindrical polar co-ordinates with $R=\sqrt{ }\left(r^{2}+z^{2}\right) ; f\left(R_{2}, z+i a, \theta\right), f\left(R_{1}, z-i a, \theta\right)$ will be complex conjugate harmonic functions which will generally be discontinuous on the plane $z=0$ when $r=a$. By taking suitable combinations of such functions, we can generate real harmonic functions satisfying the boundary conditions defined by equations $(17,19)$. Examples of the use of this method are given below and further details and examples are discussed by Green [2] and Love [8]. An integral formulation of the method for the axisymmetric case is given by Green and Zerna ([3], pp. 172-8). It is easily shown that $R^{m} P_{m}^{n}(z / R) \cos n\left(\theta+\theta_{0}\right), R^{m} Q_{m}^{n}(z / R) \cos n\left(\theta+\theta_{0}\right)$ are harmonic functions where $P, Q$ are associated Legendre functions and $m, n$ are integers or zero. These forms enable us to generate suitable functions for a wide range of problems.

\section{THE CONTACT PROBLEM}

(a) Non-axisymmetric case

We consider first the contact problem defined by equation (7) for the more interesting case in which $n \neq 0$. We suppose that $f(r), g(r)$ are particular integrals of equations $(11,12)$ respectively, which also satisfy the conditions on displacement defined by equations $(3,7)$. It follows that the 
general expressions for $\phi, \psi$ defined by equations $(11,12)$ are

$$
\begin{aligned}
& \phi(r, \theta, 0)=\left(f(r)+A r^{n}+B r^{-n}\right) \cos n\left(\theta+\theta_{0}\right), \\
& \psi(r, \theta, 0)=\left(g(r)+C r^{n}+D r^{-n}\right) \sin n\left(\theta+\theta_{0}\right),
\end{aligned}
$$

for $a \geq r$, where $A, B, C, D$ are arbitrary constants.

If the solution is to be bounded at the origin, we must have $B=D=0$, and substitution into equation (3) shows that $A+C=0$. Hence we have

$$
\left.\begin{array}{c}
\phi(r, \theta, 0)=\left(f(r)+A r^{n}\right) \cos n\left(\theta+\theta_{0}\right), \\
\psi(r, \theta, 0)=\left(g(r)-A r^{-n}\right) \sin n\left(\theta+\theta_{0}\right),
\end{array}\right\}
$$

for $a \geq r$.

By a similar argument applied to equations $(4,7,9,10)$ it follows that

$$
\left.\begin{array}{c}
\frac{\partial \phi}{\partial z}(r, \theta, 0)=\alpha(1-\nu) r^{-n} \cos n\left(\theta+\theta_{0}\right), \\
\frac{\partial \psi}{\partial z}(r, \theta, 0)=\alpha r^{-n} \sin n\left(\theta+\theta_{0}\right),
\end{array}\right\}
$$

for $r>a$, where $\alpha$ is an arbitrary constant.

We now express $\phi, \psi$ as the sum of two hrmonic functions $\phi_{1}, \phi_{2} ; \psi_{1}, \psi_{2}$ as outlined in section 4 , and substitute for $\left[\partial \Phi_{1}(r, 0)\right] / \partial z$ in equation (18) to obtain

$$
\Phi_{1}(r, 0)=\Phi(r, 0)-\Phi_{2}(r, 0)=\frac{-\alpha(1-\nu)(2 n-3) ! !}{(2 n-2) ! ! r^{n-1}}, \quad r>0
$$

where $(2 m) ! ! \equiv 2.4 .6 \ldots(2 m) ;(2 m-1) ! ! \equiv 1.3 .5 \ldots(2 m-1)$. Similarly

$$
\Psi_{1}(r, 0)=\Psi(r, 0)-\Psi_{2}(r, 0)=\frac{-\alpha(2 n-3) ! !}{(2 n-2) ! ! r^{n-1}}, \quad r>0 .
$$

The continuity condition at $r=a$ can be stated in the form

$$
\underset{r \rightarrow a}{\operatorname{Lt}}(a-r)\left(\sigma_{r z}, \sigma_{\mathrm{Ar}}\right)=0 .
$$

From equations $(4,19)$ it can be shown that this requires

$$
\underset{t \rightarrow a}{L t} \frac{\mathrm{d}}{\mathrm{d} t} \int_{0}^{t} \frac{s^{n+1}\left(\Phi_{2}(s, 0), \Psi_{2}(s, 0)\right) \mathrm{d} s}{\sqrt{ }\left(t^{2}-s^{2}\right)}=0 .
$$

Substituting for $\Phi_{2}(r, 0), \Psi_{2}(r, 0)$ from equations $(24,26,27)$ and applying these conditions we find

$$
\alpha=\frac{-2(2 n-2) ! !(U(a)+V(a)) a^{2 n-1}}{\pi(2 n-3) ! !(2-\nu)}
$$


where

$$
U(t), V(t)=t^{-2 n} \frac{\mathrm{d}}{\mathrm{d} t} \int_{0}^{t} \frac{s^{n+1}(f(s), g(s)) \mathrm{d} s}{\sqrt{ }\left(t^{2}-s^{2}\right)} .
$$

In terms of these functions, we have

$$
\begin{aligned}
\frac{\partial \Phi_{2}(r, 0)}{\partial z}= & \frac{2 r^{n-1}}{\pi} \frac{\mathrm{d}}{\mathrm{d} r} \int_{r}^{a} \frac{t}{\sqrt{ }\left(t^{2}-r^{2}\right)}\{U(t)-U(a) \\
& \left.+\frac{(1-\nu)}{(2-\nu)} 1-\left(\frac{a}{t}\right)^{2 n-1}[U(a)+V(a)]\right\} \mathrm{d} t, \\
\frac{\partial \Psi_{2}(r, 0)}{\partial z}= & \frac{2 r^{n-1}}{\pi} \frac{\mathrm{d}}{\mathrm{d} r} \int_{r}^{a} \frac{t}{\sqrt{ }\left(t^{2}-r^{2}\right)}\{V(t)-V(a) \\
& \left.+1-\left(\frac{a}{t}\right)^{2 n-1} \frac{[U(a)+V(a)]}{(2-\nu)}\right\} \mathrm{d} t,
\end{aligned}
$$

for $a \geq r$, from equation (19).

Also, from equations $(25,30)$ we have

$$
\begin{aligned}
& \frac{\partial \Phi_{1}(r, 0)}{\partial z}=\frac{-2(2 n-2) ! !(1-\nu)[U(a)+V(a)] a^{2 n-1}}{\pi(2 n-3) ! !(2-\nu) r^{n}}, \\
& \frac{\partial \Psi_{1}(r, 0)}{\partial z}=\frac{-2(2 n-2) ! ![U(a)+V(a)] a^{2 n-1}}{\pi(2 n-3) ! !(2-\nu) r^{n}},
\end{aligned}
$$

for all $r$.

Hence, the distribution of tangential surface traction can be obtained from the sum of corresponding terms in these equations, using equation (4).

\section{(b) Axisymmetric case}

The axisymmetric problem $(n=0)$ can be solved by other, more direct, methods, but we give a solution here for the sake of completeness. When $n=0$, the boundary conditions on $\phi, \psi$ expressed by equations $(24,25)$ are replaced by

$$
\left.\begin{array}{rlrl}
\phi(r, \theta, 0) & =f(r)+A, & & a \geq r ; \\
\psi(r, \theta, 0) & =g(r)+B, & & a \geq r ; \\
\frac{\partial \phi}{\partial z}(r, \theta, 0) & =\frac{\partial \psi}{\partial z}(r, \theta, 0)=0, & & r \geq a .
\end{array}\right\}
$$

The two functions $\phi, \psi$ are therefore independent and the solution for each can follow that given by Green [2], the constants $A, B$ being determined by requirements of continuity at $r=a$.

The final results corresponding to equation (32) are

for $a \geq r$.

$$
\left.\begin{array}{l}
\frac{\partial \phi}{\partial z}(r, \theta, 0)=\frac{\partial \Phi(r, 0)}{\partial z}=\frac{2}{\pi r} \frac{\mathrm{d}}{\mathrm{d} r} \int_{r}^{a} \frac{t}{\sqrt{ }\left(t^{2}-r^{2}\right)}[U(t)-U(a)] \mathrm{d} t, \\
\frac{\partial \psi}{\partial z}(r, \theta, 0)=\frac{\partial \Psi(r, 0)}{\partial z}=\frac{2}{\pi r} \frac{\mathrm{d}}{\mathrm{d} r} \int_{r}^{a} \frac{t}{\sqrt{ }\left(t^{2}-r^{2}\right)}[V(t)-V(a)] \mathrm{d} t,
\end{array}\right\}
$$


(c) Example Mindlin's problem

To illustrate the use of the method and to check the results obtained in section 5(a), we consider the problem defined by the boundary conditions

$$
\begin{array}{rlrl}
u_{x} & =u_{0} ; u_{y}=0 ; & z=0, & a \geq r ; \\
\sigma_{x z} & =\sigma_{y z}=0 ; & z=0, & r>a ; \\
\sigma_{z z}=0 ; & z=0, & \text { all } r,
\end{array}
$$

where $u_{0}$ is a constant.

A solution to this problem was first given by Mindlin[9] using a different method.

The surface displacements within $a \geq r$ could be represented by the potential functions

$$
\phi(r, \theta, 0)=u_{0} r \cos \theta, \psi(r, \theta, 0)=0,
$$

corresponding to the particular integrals

$$
f(r)=u_{0} r ; g(r)=0
$$

of equations $(11,12)$, with $n=1$.

Substitution in equation (31) gives

$$
U(t)=2 u_{0} ; V(t)=0,
$$

and hence from equations $(32,33)$, we have

$$
\begin{aligned}
\frac{\partial \Phi(r, 0)}{\partial z} & =\frac{\partial \Phi_{2}(r, 0)}{\partial z}+\frac{\partial \Phi_{1}(r, 0)}{\partial z} \\
& =\frac{4 u_{0}(1-\nu)}{\pi(2-\nu)} \frac{\mathrm{d}}{\mathrm{d} r} \int_{r}^{a} \frac{(t-a) \mathrm{d} t}{\sqrt{ }\left(t^{2}-r^{2}\right)}-\frac{4 u_{0}(1-\nu) a}{\pi(2-\nu) r} \\
& =\frac{4 u_{0}(1-\nu)}{\pi(2-\nu)}\left(\frac{\sqrt{ }\left(a^{2}-r^{2}\right)-a}{r}\right),
\end{aligned}
$$

for $a \geq r$.

By the same method we obtain

$$
\frac{\partial \Psi(r, 0)}{\partial z}=\frac{4 u_{0}}{\pi(2-\nu)}\left(\frac{\sqrt{ }\left(a^{2}-r^{2}\right)-a}{r}\right)
$$

for $a \geq r$, whilst in the region $r>a$, we have

$$
\begin{aligned}
& \frac{\partial \Phi(r, 0)}{\partial z}=-\frac{4 u_{0} a(1-\nu)}{\pi r(2-\nu)}, \\
& \frac{\partial \Psi(r, 0)}{\partial z}=-\frac{4 u_{0} a}{\pi r(2-\nu)}
\end{aligned}
$$


Substituting these values into equation (4), we obtain

$$
\sigma_{x z}=-\frac{4 u_{0} G}{\pi(2-\nu) \sqrt{ }\left(a^{2}-r^{2}\right)} ; \quad \sigma_{y z}=0,
$$

for the surface tractions in $a \geq r$, agreeing with the results of Mindlin[9].

To find the stresses and displacements in the body of the solid, we need to find harmonic functions $\Phi(r, z) \cos \theta, \Psi(r, z) \sin \theta$ satisfying equations (40-42) on $z=0$.

The function $(r-z) \cos \theta / r$ is harmonic and continuous in $z>0$ and in conjunction with the results of section (4) it suggests the form

$$
\begin{aligned}
\frac{\partial \phi}{\partial z} & =\frac{2 u_{0}(1-\nu) i}{\pi(2-\nu)}\left(R_{1}-z+i a-R_{2}+z+i a\right) \frac{\cos \theta}{r} \\
& =\frac{2 u_{0}(1-\nu)}{\pi(2-\nu)}\left[i\left(R_{2}-R_{1}\right)-2 a\right] \frac{\cos \theta}{r}
\end{aligned}
$$

which satisfies the boundary conditions on $z=0$, in view of equation (22).

A suitable integral of equation (44) which satisfies conditions of continuity on $r=0$ and at infinity is

$$
\begin{aligned}
\phi= & \frac{u_{0}(1-\nu)}{\pi(2-\nu)}\left[i r \log \left(\frac{R_{1}+z-i a}{R_{2}+z+i a}\right)+\frac{i(z-i a) R_{1}}{r}\right. \\
& \left.-\frac{i(z+i a) R_{2}}{r}+\frac{4 z a}{r}\right] \cos \theta
\end{aligned}
$$

The corresponding expression for $\psi$ is

$$
\psi=\frac{u_{0}}{\pi(2-\nu)}\left[i r \log \left(\frac{R_{1}+z-i a}{R_{2}+z+i a}\right)+\frac{i(z-i a) R_{1}}{r}-\frac{i(z+i a) R_{2}}{r}+\frac{4 z a}{r}\right] \sin \theta .
$$

Expressions for stresses and displacements can now be obtained by substituting in equations $(1,2)$, using the results of section 4 to express $\phi, \psi$ in real form.

\section{THE CRACK PROBLEM}

(a) Non-axisymmetric case

The solution of the crack problem for $n \neq 0$ follows the same pattern as that of the corresponding contact problem (section 5(a)). However, extra arbitrary functions appear in the general solution of equations $(16,17)$, which have to be determined by more stringent conditions of continuity on $\mathscr{F}(r, 0), \mathscr{G}(r, 0)$ at $r=a$.

If $f(r), g(r)$ are particular integrals of equations $(16,17)$ respectively, which also satisfy the conditions on stress imposed by equations $(4,13)$, we have the general solution

$$
\begin{aligned}
& \mathscr{F}(r, 0)=f(r)+A r^{n}+B(1-\nu) r^{n+2} \\
& \mathscr{G}(r, 0)=g(r)+C r^{n}-B r^{n+2}
\end{aligned}
$$

for $a \geq r$, corresponding to equation (24) of the contact problem solution. 
Also,

$$
\frac{\partial \mathscr{F}(r, 0)}{\partial z}=\frac{\partial \mathscr{G}(r, 0)}{\partial z}=\alpha r^{-n}
$$

for $r>a$, from equations $(3,10,13,15) . A, B, C, \alpha$ are arbitrary constants to be determined from conditions of continuity at $r=a$.

Expressing $\mathscr{F}(r, 0), \mathscr{G}(r, 0)$ each as the sum of two functions $\mathscr{F}_{1}, \mathscr{F}_{2}, \mathscr{G}_{1}, \mathscr{G}_{2}$ as in section 5(a) we obtain

$$
\mathscr{F}_{1}(r, 0)=\mathscr{G}_{1}(r, 0)=-\frac{\alpha(2 n-3) ! !}{(2 n-2) ! ! r^{n-1}} ; \quad r>0
$$

from equation (18).

In the crack problem, the displacements $u_{r}, u_{0}$ must tend to zero as $\mathbf{r}$ approaches a from below and hence we must have

$$
\left.\begin{array}{c}
\underset{r \rightarrow a^{-}}{\operatorname{Lt}}\left(\frac{\partial \mathscr{F}_{2}(r, 0)}{\partial z}, \frac{\partial \mathscr{G}_{2}(r, 0)}{\partial z}\right)=0 \\
\underset{r \rightarrow a^{-}}{\operatorname{Lt}}\left(\frac{\partial^{2} \mathscr{F}_{2}(r, 0)}{\partial r \partial z}, \frac{\partial^{2} \mathscr{G}_{2}(r, 0)}{\partial r \partial z}\right)=0
\end{array}\right\}
$$

from equations $(4,14)$.

These four conditions enable us to find the four arbitrary constants in equations $(47,48)$ and hence to complete the solution for $\mathscr{F}_{2}(r, 0), \mathscr{G}_{2}(r, 0)$ from equation (19).

The final solution is

$$
\begin{aligned}
\frac{\partial \mathscr{F}(r, 0)}{\partial z}= & \alpha r^{-n}+\frac{2 r^{n-1}}{\pi} \frac{\mathrm{d}}{\mathrm{d} r} \int_{r}^{a} \frac{t}{\sqrt{ }\left(t^{2}-r^{2}\right)}[U(t)-U(a) \\
& +\frac{a\left[U^{\prime}(a)-V^{\prime}(a)\right](1-\nu)\left[1-\left(t^{2} / a^{2}\right)\right]}{2(2-\nu)} \\
& \left.+\frac{a\left[U^{\prime}(a)+(1-\nu) V^{\prime}(a)\right](a / t)^{2 n-1}-1}{(2 n-1)(2-\nu)}\right] \mathrm{d} t \\
\frac{\partial \mathscr{G}(r, 0)}{\partial z}= & \alpha r^{-n}+\frac{2 r^{n-1}}{\pi} \frac{\mathrm{d}}{\mathrm{d} r} \int_{r}^{a} \frac{t}{\sqrt{ }\left(t^{2}-r^{2}\right)}[V(t)-V(a) \\
& +\frac{a\left[V^{\prime}(a)-U^{\prime}(a)\right]\left[1-\left(t^{2} / a^{2}\right)\right]}{2(2-\nu)} \\
& \left.+\frac{a\left[U^{\prime}(a)+(1-\nu) V^{\prime}(a)\right](a / t)^{2 n-1}-1}{(2 n-1)(2-\nu)}\right] \mathrm{d} t
\end{aligned}
$$

for $a \geq r$, where

$$
\alpha=\frac{2(2 n-2) ! ! a^{2 n}\left[U^{\prime}(a)+(1-\nu) V^{\prime}(a)\right]}{\pi(2 n-1) ! !(2-\nu)}
$$

and $U(t), V(t)$ are defined by equation (31). 
(b) Axisymmetric case

When $n=0$, the boundary conditions on $\mathscr{F}(r, z), \mathscr{G}(r, z)$ comparable with equations $(47,48)$ take the form

$$
\begin{aligned}
& \left.\begin{array}{l}
\mathscr{F}(r, 0)=f(r)+A r^{2}+B ; \\
\mathscr{G}(r, 0)=g(r)+C r^{2}+D ;
\end{array}\right\} a \geq r, \\
& \frac{\partial \mathscr{F}(r, 0)}{\partial z}=\frac{\partial \mathscr{G}(r, 0)}{\partial z}=0 ; \quad r>a .
\end{aligned}
$$

We note that the two functions are independent as in the corresponding contact problem. We therefore apply Green's solution to each function separately, using the continuity conditions of equation (50) to find the four arbitrary constants.

The final solution is

$$
\begin{aligned}
\frac{\partial \mathscr{F}(r, 0)}{\partial z}= & \phi(r, \theta, 0)=-\frac{2}{\pi r} \frac{\mathrm{d}}{\mathrm{d} r} \int_{r}^{a} \frac{t}{\sqrt{ }\left(t^{2}-r^{2}\right)}[U(t)-U(a) \\
& \left.+\frac{U^{\prime}(a)}{2 a}\left(a^{2}-t^{2}\right)\right] \mathrm{d} t, \\
\frac{\partial \mathscr{G}(r, 0)}{\partial z}= & \psi(r, \theta, 0)=-\frac{2}{\pi r} \frac{\mathrm{d}}{\mathrm{d} r} \int_{r}^{a} \frac{t}{\sqrt{ }\left(t^{2}-r^{2}\right)}[V(t)-V(a) \\
& \left.+\frac{V^{\prime}(a)}{2 a}\left(a^{2}-t^{2}\right)\right] \mathrm{d} t,
\end{aligned}
$$

for $a \geq r$.

(c) Example: the penny-shaped crack in shear

The most straightforward example of a non-axisymmetric problem is that of the penny-shaped crack in shear, defined by the boundary conditions

$$
\begin{gathered}
u_{x}=u_{y}=0, r>a, z=0, \\
\sigma_{x z}=p_{0} ; \sigma_{y z}=0 ; a \geq r, z=0, \\
\sigma_{z z}=0 ; \text { all } r, z=0:
\end{gathered}
$$

The surface tractions within $a \geq r$ could be represented by the potential functions

$$
\frac{\partial \phi}{\partial z}(r, \theta, 0)=\frac{(1-\nu) p_{0} r \cos \theta}{G} ; \frac{\partial \psi}{\partial z}(r, \theta, 0)=0 .
$$

corresponding to the particular integrals

$$
f(r)=-\frac{(1-v) p_{0} r^{3}}{8 G} ; g(r)=0
$$

of equations $(16,17)$ with $n=1$. 
Substitution in equation (31) gives

$$
U(t)=-\frac{(1-\nu) p_{0} t^{2}}{3 G} ; V(t)=0,
$$

and hence from equations $(51,52)$

$$
\begin{aligned}
\phi(r, \theta, 0) & =\frac{\partial \mathscr{F}(r, 0) \cos \theta}{\partial z}=\frac{4(1-\nu) p_{0} \cos \theta}{3 \pi G(2-\nu)}\left[\frac{\left(a^{2}-r^{2}\right)^{3 / 2}-a^{3}}{r}\right] ; a \geq r, \\
& =-\frac{4(1-\nu) p_{0} a^{3} \cos \theta}{3 \pi G(2-\nu) r} ; r>a \\
\psi(r, \theta, 0) & =\frac{\partial G(r, 0) \sin \theta}{\partial z}=\frac{4(1-\nu) p_{0} \sin \theta}{3 \pi G(2-\nu)}\left[\frac{\left(a^{2}-r^{2}\right)^{3 / 2}-a^{3}}{r}\right] ; a \geq r . \\
& =-\frac{4(1-\nu) p_{0} a^{3} \sin \theta}{3 \pi G(2-\nu) r} ; r>a .
\end{aligned}
$$

Substituting into equation (3) we obtain the corresponding surface displacements

$$
u_{x}=-\frac{4(1-\nu) p_{0} \sqrt{ }\left(a^{2}-r^{2}\right)}{\pi G(2-\nu)} ; u_{y}=0 ; a \geq r,
$$

which tend to zero as required as $r$ approaches $a$.

To find the values of $\phi, \psi$ throughout the solid and hence the complete stress and displacement field, we use the method outlined in section 4 and exemplified in section 5(c). It is easily verified that the real harmonic functions

$$
\phi=\omega(r, z) \cos \theta ; \psi=\omega(r, z) \sin \theta
$$

where

$$
\begin{aligned}
\omega(r, z)= & \frac{p_{0}(1-\nu)}{\pi G(2-\nu)}\left[i r z \log \left(\frac{R_{2}+z+i a}{R_{1}+z-i a}\right)+\frac{i\left(R_{2}^{3}-R_{1}^{3}\right)}{3 r}\right. \\
& \left.-i r\left(R_{2}-R_{1}\right)+a(z+i a) \frac{R_{2}}{r}+a(z-i a) \frac{R_{1}}{r}+\frac{4 a^{3}}{3 r}\right]
\end{aligned}
$$

satisfy the boundary conditions defined by equation (58) and are bounded at infinity and on $r=0$ for $z \geq 0$. Equation (61) therefore defines the solution of our problem, from which particular expressions for stresses and displacements can be obtained using equations $(1,2)$.

(d) Second example: the penny-shaped crack in torsion about a diametral axis

As an example of the application of the method in cases where $n>1$, we consider a solid in torsion about an axis which is a diameter of a penny-shaped crack. This case should be distinguished from the axisymmetric torsion problem (discussed for example by Weinstein [10]) in which the axis of torsion is perpendicular to the crack plane.

We suppose the axis of torsion to be the $x$-axis, in which case the stresses on the plane $z=0$ 
in an unflawed solid would be

where $c$ is a constant.

$$
\sigma_{x z}=-c y ; \sigma_{y z}=0 ; \sigma_{z z}=0,
$$

To make the surfaces of the crack stress free, we need to superpose the stress system defined by the boundary conditions

$$
\begin{gathered}
u_{x}=u_{y}=0 ; r>a, z=0, \\
\sigma_{x z}=c y ; \sigma_{y z}=0 ; a \geq r, z=0, \\
\sigma_{z z}=0 ; \text { all } r, z=0 .
\end{gathered}
$$

The surface traction on $a \geq r$ could be represented by the potential functions

$$
\frac{\partial \phi}{\partial z}(r, \theta, 0)=0 ; \frac{\partial \psi}{\partial z}(r, \theta, 0)=\frac{c r^{2}(1-\cos 2 \theta)}{4 G} .
$$

This expression contains axisymmetric and non-axisymmetric components which must be treated separately using the resuly of sections $6(a, b)$.

For the axisymmetric term, a suitable particular integral of equation (17) is

and hence

$$
g(r)=-\frac{c r^{4}}{64 G}
$$

$$
\begin{aligned}
\psi(r, \theta, 0)=\frac{\partial \mathscr{G}(r, 0)}{\partial z} & =-\frac{2 c\left(a^{2}-r^{2}\right)^{3 / 2}}{9 \pi G} ; a \geq r, \\
& =0 ; r>a,
\end{aligned}
$$

from equations $(31,54,66)$.

The non-axisymmetric term in equation (65) can be accommodated within the framework of equation (13) by taking $n=2, \theta_{0}=\pi / 4$, in which form suitable particular integrals of equations $(16,17)$ are

$$
f(r)=0 ; g(r)=\frac{c r^{4}}{48 G}
$$

Substituting in equations $(31,51,52)$ we obtain the solution

$$
\begin{aligned}
& \phi(r, \theta, 0)=-\frac{\partial \mathscr{F}(r, 0)}{\partial z} \sin 2 \theta=\frac{c(1-\nu) \omega_{1}(r, 0) \sin 2 \theta}{60 \pi G(2-\nu)} \\
& \psi(r, \theta, 0)=\frac{\partial G(r, 0)}{\partial z} \cos 2 \theta=\frac{-c(1-\nu) \omega_{1}(r, 0) \cos 2 \theta}{60 \pi G(2-\nu)}
\end{aligned}
$$

where

$$
\begin{aligned}
\omega_{1}(r, 0) & =\frac{16}{3}\left\{\frac{2\left[a^{5}-\left(a^{2}-r^{2}\right)^{5 / 2}\right]}{r^{2}}-5\left(a^{2}-r^{2}\right)^{3 / 2}\right\} ; a \geq r, \\
& =\frac{32 a^{5}}{3 r^{2}} ; r>a .
\end{aligned}
$$


The complete solution is then obtained by adding equations $(67,69)$. Substitution in equation (4) gives the total surface displacements

for $a \geq r$.

$$
\left.\begin{array}{c}
u_{*}=\frac{2 c(4-3 \nu) y \sqrt{ }\left(a^{2}-r^{2}\right)}{3 \pi G(2-\nu)} \\
u_{y}=\frac{-2 c \nu(1-\nu) x \sqrt{ }\left(a^{2}-r^{2}\right)}{3 \pi G(2-\nu)}
\end{array}\right\}
$$

To find the stresses and displacements in the body of the solid, we note that the real harmonic functions

$$
\begin{aligned}
& \phi(r, \theta, z)=\frac{c(1-\nu) \omega_{1}(r, z) \sin 2 \theta}{60 \pi G(2-\nu)} \\
& \psi(r, \theta, z)=\frac{c \omega_{2}(r, z)}{12 \pi G}-\frac{c(1-\nu) \omega_{1}(r, z) \cos 2 \theta}{60 \pi G(2-\nu)}
\end{aligned}
$$

satisfy the boundary conditions obtained from the sum of equations $(67,69)$ and appropriate continuity conditions if

$$
\begin{aligned}
\omega_{1}(r, z)= & 15 i r^{2} z \log \left(\frac{R_{2}+z+i a}{R_{1}+z-i a}\right)+2 i \frac{\left(R_{2}^{5}-R_{1}^{5}\right)}{r^{2}} \\
& +5 i\left(R_{2}^{3}-R_{1}^{3}\right)\left(1-\frac{8 a^{2}}{3 r^{2}}\right)+15 i\left(a^{2}-r^{2}\right)\left(R_{2}-R_{1}\right) \\
& +15 a z\left(R_{2}+R_{1}\right)+\frac{10 a}{r^{2}}\left[(z+i a) R_{2}^{3}+(z-i a) R_{1}^{3}\right]+\frac{32 a^{5}}{3 r^{2}}, \\
\omega_{2}(r, z)= & i z\left(3 r^{2}-2 a^{2}-2 z^{2}\right) \log \left(\frac{R_{2}+z+i a}{R_{1}+z-i a}\right) \\
& +\frac{11 i}{3}\left(R_{2}^{3}-R_{1}^{3}\right)+5 i\left(a^{2}-r^{2}\right)\left(R_{2}-R_{1}\right) \\
& +9 a z\left(R_{2}+R_{1}\right) .
\end{aligned}
$$

These results therefore define the solution to the problem.

\section{CONCLUSION}

The solution described in this paper, taken in conjunction with Green's solution for the penny-shaped crack opened by an arbitrary normal pressure [2], provides a general solution to the problem of the penny-shaped crack which has the advantage of mathematical simplicity in comparison with other methods of solution. We also note that the general solution of section 2 is capable of application to other classes of surface loading problem involiving tangential tractions and displacements.

\section{REFERENCES}

(11 J. R. BARBER, Int. J. Mech. Sic. 13, 727 (1974).

[2] A. E. GREEN, Proc. Camb. Phil. Soc. 45, 251 (1949).

[3] R. A. WESTMANN, J. appl. Mech. 32, 411 (1965). 
[4] R. MUKI, Prog. Solid Mech. 1, 399 (1960).

[5] M. LOWENGRUB and I. N. SNEDDON, Int. J. Engng Sci. 10, 899 (1972).

[6] A. E. GREEN and W. ZERNA, Theoretical Elasticity. Clarendon Press (1954).

[7] E. T. COPSON, Proc. Edinburgh Math. Soc. 8, 14 (1947).

[8] A. E. H. LOVE, Q. J. Math. 10, 161 (1939).

[9] R. D. MINDLIN, J. appl. Mech. 16, 259 (1949).

[10] A. WEINSTEIN, Q. J. Appl. Math. 10, 77 (1952).

(Received 22 March 1974) 